\title{
Imaginario de ciudad de la comunidad Intillay Zhysua, a partir de la recuperación de la cultura Mhuysqa*
}

\author{
City Perception of the Intillay Zhysua Community, \\ from the Recuperation of the Mhuysqa Culture
Imaginário de cidade da comunidade Intillay Zhysua, desde a recuperação da cultura Mhuysqa

Recibido julio 9 de 2018. Aceptado abril 26 de 2019.

Edna Alejandra León Espinosa**
https://orcid.org/0000-0001-8157-9912

Colombia

, Para citar este artículo:

León-Espinosa, Edna-Alejandra (2019). Imaginario de ciudad de la comunidad Intillay Zhysua, a partir de la recuperación de la cultura Mhuysqa. Ánfora, 26(47),

61-86. DOI:

https://doi.org/10.30854/anfv26.n47.2019.633

Universidad Autónoma de Manizales. ISSN 0121-6538/ e-ISSN 2248-6941

\section{Resumen}

Objetivos: determinar el imaginario de ciudad de la comunidad Intillay Zhysua, a partir de la integración de saberes no científicos y originarios como elementos constitutivos de los órdenes sociales emergentes. Se interpreta la realidad mediada por la recuperación y resignificación de la Cultura Mhuysqa, en una configuración imaginaria y representacional de la ciudad de Bogotá, según la comunidad Intillay Zhysua. Metodología: el estudio se ubica en los enfoques cualitativos de la Investigación Social, fundamentada en una perspectiva interpretativaque emplea como método investigativo la etnografía apoyada en la observación

\footnotetext{
* Este artículo es resultado del proyecto de investigación en la Maestría en Investigación Social Interdisciplinaria inscrita en la Facultad de Ciencias y Educación de la Universidad Distrital Francisco José de Caldas. Bogotá, Colombia. Investigación iniciada en enero del 2015 y finalizada en noviembre de 2016.

** Magíster en Investigación. Licenciada en Ciencias Sociales. Docente de la Universidad Distrital Francisco José de Caldas y de la Corporación para la Educación Superior CES. Correo:alejandraleon.mg@gmail.com
} 
León-Espinosa, Edna-Alejandra (2019). Imaginario de ciudad de la comunidad Intillay Zhysua, a partir de la recuperación de la cultura Mhuysqa. Ánfora, 26(47), 61-86. Universidad Autónoma de Manizales.

participante. Se optó por técnicas de recolección de información como diarios de campo, entrevistas y análisis documental. Dadas las pautas del diseño metodológico, se realizó la triangulación de las técnicas de registro señaladas, como una estrategia que permitió tener una visión completa de la realidad social investigada. Resultados: se halló la existencia de discrepancias sobre el imaginario instituido de ciudad creado hegemónicamente y el imaginario que ha consolidado la comunidad Intillay Zhysua. El estudio también revela una configuración imaginaria instituyente de ciudad, fundada en la recuperación de los sustentos cosmogónicos y los aportes éticos que ofrece la cultura Mhuysqa, a partir de experiencias subjetivas que hacen efectiva una nueva representación de la realidad. Conclusiones: se constituye una perspectiva reflexiva y crítica con respecto a la subordinación de saberes, que valora las maneras en que los sujetos interpretan y significan su existencia al construir un conocimiento constitutivo de la realidad social orientada hacia el reconocimiento de la diversidad del ser humano.

Palabras-clave: Imaginario; Ciudad; Cultura Mhuysqa; Resignificación; Saberes originarios; Identidad.

\section{Abstract}

Objective: to determine the city perception of the Intillay Zhysua community, based on the integration of non-scientific and native knowledge as constitutive elements of emergent social orders. The mediated reality is interpreted through the recuperation and resignification of the Mhuysqa Culture, in a perceptual and representational configuration of the city of Bogotá, according to the Intillay Zhysua community. Methodology: the study focuses on qualitative analysis of social research, based on an interpretative perspective that employs ethnographic investigative methods founded on participative observation. Information gathering techniques such as field diaries, interviews and documentary analysis were utilized. Adhering to the methodological design, triangulation of the abovementioned research techniques was carried out, as a strategy to obtain a complete overview of the social reality being investigated. Results: the findings show discrepancies between the created hegemonic city perception and the consolidated perception of the Intillay Zhysua community. The study also reveals a perception instituting configuration of the city founded on the recuperation of the cosmogonic concepts and ethical contributions offered by the Mhuysqa culture, based on subjective experiences that effectuate a new representation of reality. Conclusions: a reflexive and critical perspective is constituted with respect to the subordination of knowledge, which places values in the ways in which subjects interpret and assign meaning to their existence by constructing a constitutive knowledge of social reality oriented towards the recognition of diversity of the human being. 
Keywords: Perception; City; Mhuysqa Culture; Resignification; Native knowledge; Identity.

\section{Resumo}

Objetivo: determinar o imaginário de cidade da comunidade Intillay Zhysua, a partir da integração do conhecimento não científico e originário como elementos constitutivos das ordens sociais emergentes. A realidade mediada é interpretada pela recuperação e ressignificação daCultura Mhuysqa,emumaconfiguração imagináriae representacional da cidade de Bogotá, segundo a comunidade Intillay Zhysua. Metodologia: o estudo está localizado nas abordagens qualitativas da Pesquisa Social, baseado em uma perspectiva interpretativa que utiliza a etnografia baseada na observação participante como método de pesquisa. Optou-se por técnicas de coleta de informações, como diários de campo, entrevistas e análise documental. Dadas as diretrizes do desenho metodológico, foi realizada a triangulação das técnicas de registro indicadas, como estratégia que permitiu ter uma visão completa da realidade social investigada. Resultados: constatou-se a existência de discrepâncias acerca do imaginário instituído de cidade hegemonicamente criada, e do imaginário que consolidou a comunidade Intillay Zhysua. O estudo também revela uma configuração instituinte imaginária da cidade, baseada na recuperação dos modos de vida cosmogônicos e nas contribuições éticas oferecidas pela cultura Mhuysqa, baseadas em experiências subjetivas que tornam efetiva uma nova representação da realidade. Conclusões: constitui-se uma perspectiva reflexiva e crítica em relação à subordinação do conhecimento, que valoriza as formas pelas quais os sujeitos interpretam e significam sua existência, construindo um conhecimento constitutivo da realidade social orientada para o reconhecimento da diversidade do ser humano.

Palavras-chave: Imaginário; Cidade; Cultura Mhuysqa; Ressignificação; Conhecimento originário; Identidade. 


\section{Introducción}

El mundo está formado por un conjunto de culturas, cada una con singularidades y atributos e, incluso, contrapuestas o incompatibles entre sí. No obstante la occidental, una entre tantas, tiene un papel destacado al imponer su estilo de vida y patrones de dominación que tienden a transformar y alterar las particularidades de las culturas.

Ante este panorama se presentan experiencias colectivas que aspiran a construir otras posibilidades de existencia al incorporar, en la práctica cotidiana, saberes de las culturas originarias ${ }^{1}$, a la vez que se expresa un distanciamiento crítico de la versión occidental de progreso y con el que históricamente se han configurado las ciudades como "el corazón de la reproducción de los modos de vida dominantes, coloniales, modernos, capitalistas” (Rodríguez, 2013, p. 226).

En tal distanciamiento, se distingue que los aspectos culturales exógenos provienen de un proceso de invasión que implicó su imposición en la cultura y, con ello, un proceso de asimilación que, como señala Echeverría (2011), propicia diferentes tipos de interacción que desatan transformaciones en unos casos constructivas e ineludiblemente destructivas en otros. Lo anterior no ha de significar, o no necesariamente, que con la asimilación desaparezca toda diversidad.

Se plantea entonces, un ejercicio investigativo que analiza el proceso social, a partir del cual se forma la comunidad Intillay Zhysua y crea mecanismos de significación cultural en zonas urbanas. Se analiza la configuración imaginaria de los sujetos, la manera en que perciben su vida en sociedad desvelando las significaciones y configuraciones forjadas, así como los discursos que se tejen en torno a las maneras de ver la realidad.

Conforme a ello, será necesario comprender la cultura como el proceso en que una organización o comunidad forja su identidad, su forma singular y concreta de ser, dado que, como menciona Echeverría (2011), la dimensión cultural de la existencia social estaría dada por el hecho de que en cada acto de la cotidianidad, el ser humano cultiva la dimensión identitaria de su existencia (p. 415). Y al hablar de identidad, ésta debe ser entendida como el punto de enlace entre los discursos y prácticas que intentan interpelar por una particular filiación de los individuos y por los procesos que ocasionan subjetividades y constituyen a los sujetos que se identifican o no con esas filiaciones (Hall; citado por Restrepo, 2004, p. 57).

\footnotetext{
1 Término comprendido a partir de las investigaciones de Fernando Huanacuni Mamani cuyo entendimiento parte del reconocimiento de las culturas originarias como los Navajo, Lakota, Maya, Kuna, Mhuysqa, Kogui, Arhuacos, Guaraníes, Aymaras, entre muchos otros, que guardan la tradición ancestral y recuperan, mantienen las formas antiguas de vida y relacionamiento que han permitido el respeto y cuidado del equilibrio de la Tierra (Huanacuni, 2015, pp. 15-21).
} 
Este trabajo de investigación también se establece bajo una perspectiva analítica de la ciudad, para comprender el tejido social y cultural que se presenta en los grupos que la habitan y entrever cómo determinadas acciones hacen parte de un imaginario que le confiere nuevos sentidos y significaciones a ésta. Es precisamente en la emergencia de un nuevo imaginario sobre la ciudad donde unas acciones y saberes apropiados darán cuenta de dicho constructo; esto, porque "lo que da sentido a las prácticas es el imaginario social” (Taylor, 2004, p. 139). Es decir, la posibilidad de trasformación a partir de un nuevo principio de legitimación, sustentado en unas prácticas que pueden hacer efectiva una nueva perspectiva de la ciudad.

\section{¿Por qué pensar la ciudad?}

La ciudad en tanto territorio atravesado por la vivencia de quienes lo constituyen, es el escenario propicio para leer y analizar las formas de interacción social, pues como menciona Zambrano (2000), "toda ciudad se explica por su territorio y toda ciudad se entiende, si se entiende su territorio” (p. 135). De modo que en el territorio, como espacio geográfico en que se desenvuelven las relaciones sociales, se observa el mundo como sustento imaginario y representacional, al constituirse como "una construcción social" que cohesiona y orienta la vida de los sujetos (Montañez, 2001, p. 22). Es necesariamente a partir de las relaciones sociales que se producen sistemas de representaciones y se crean unas maneras colectivas de pensar, en las que determinada colectividad designa su identidad cuando elabora una representación de sí misma, fijando un imaginario, un modelo de ser.

Es por ello que la ciudad como territorio apropiado es socialmente construida. De ahí que se examine la ciudad desde sus construcciones, distribución e historia pasando por lo ideal, lo descubierto, lo deseado, pues una ciudad es una "suma de opciones de espacios, desde lo físico, a lo abstracto y figurativo, hasta lo imaginario" (Silva, 2006, p. 55); es decir, que entender la ciudad como escenario de efecto imaginario, es comprender que allí se generan producciones de sentido como evidencia de la forma en que los sujetos construyen desde sus evocaciones y deseos, modos colectivos de vivir y trasformar el lugar que habitan.

De acuerdo con este interés investigativo se analiza la comunidad Intillay Zhysua $^{2}$, conformada hace 15 años en Bogotá, con el propósito de recuperar y mantener conocimientos de los pueblos originarios. Entre 1994 y 19963, los integrantes de la comunidad eran estudiantes de las universidades públicas de la ciudad: U. Peda-

\footnotetext{
2 En lengua Quechua Inti: Sol Ilay: Mío = Intillay: Sol Mío. En lengua Mhuysqa Zhy: Mío Sua: Sol = Intillay Zhysua: Sol Mío, Sol Mío.

3 Datos suministrados por los fundadores de Intillay Zhysua.
} 
gógica Nacional y U. Distrital Francisco José de Caldas. En estos años los pueblos U’wa de la Sierra Nevada del Cocuy y Emberá Katío del Alto Sinú, se movilizaban en protestas, debido a la intromisión y extracción de recursos por multinacionales en sus territorios, situación que propició el contacto de estos estudiantes con dichos pueblos. Lo anterior se consolida como el inicio de diversos procesos en los que la comunidad se vinculará.

En 1998, se construyó en el Jardín Botánico de Bogotá una Maloca -Casa sagrada de los indígenas Huitotos del Amazonas- en que pueblos originarios, los universitarios y otras personas, llevaron a cabo encuentros en los que se apropiaban prácticas como círculos de la palabra ${ }^{4}$. De igual manera, en 2002 la comunidad se vinculó a procesos de recuperación cultural del Resguardo Mhuysqa de Cota, Cundinamarca; en 2008, fueron invitados por los pueblos originarios de Bolivia a participar del $5^{\circ}$ Encuentro Internacional de Culturas Andinas, con los cuales, actualmente, continúan asociados. En 2012, también participaron en actividades del Cabildo Mhuysqa de Bosa, entre otras, alianzas, vinculaciones y participaciones. Así, Intillay Zhysua se relacionó con entidades y organizaciones en torno a procesos de recuperación de prácticas originarias y el fortalecimiento de la cultura.

\section{Metodología}

El estudio examina la realidad social con respeto a la configuración imaginaria desde los enfoques cualitativos de la investigación social, orientados a entender los contextos que configuran lo humano y acceden a las concepciones y actuaciones de los sujetos (Taylor y Bogdan; citados por Sandoval, 2002). Por eso, la metodología se funda en una perspectiva interpretativa que emplea como método investigativo la etnografía sustentada a partir de:

1. Interpretación y comprensión de los modos de ser y actuar de los actores sociales

2. Conocimiento del sentido y los significados que los sujetos confieren a sus experiencias, visibilizando patrones de comportamiento.

Lo anterior privilegia un trabajo de campo que inició con la participación en el escenario socio-cultural de la comunidad. Esta observación participante fue la principal herramienta siguiendo algunas pautas de análisis como Caracterización del escenario socio-cultural, Identificación de los comportamientos y

4 Ritual o práctica cultural de comunicación colectiva vivida por diversos pueblos originarios. 
acciones de los sujetos e Identificación y descripción de las interacciones entre actores.

Durante este proceso investigativo se identificó un espacio llamado Kuqa (Centro de enseñanza) el cual sirvió como la unidad de análisis central para comprender las dinámicas de la comunidad y registrar lo concerniente a dos prácticas en particular. Lo anterior tuvo como propósito interpretar las vivencias desde de la observación participante, apoyada del registro continuo en diarios de campo de lo acontecido. También se emplean técnicas de recolección de información como entrevistas semi-estructuradas de preguntas abiertas y flexibles.

La comunidad está conformada por 20 personas, algunas de ellas de reciente ingreso a la comunidad. Por eso, las entrevistas se realizaron a quienes han permanecido por más de 8 años vinculados; personas con diferentes actividades y profesiones, de distinto sexo y con un rango entre 18 y 50 años. Las entrevistas requirieron la realización de varias sesiones con la misma persona y la utilización de preguntas generadoras que permitieran establecer un diálogo con base en las siguientes pautas:

1. ¿Cuál es el propósito de la comunidad?

2. ¿Cuál es su percepción sobre Bogotá?

3. ¿Cómo recuperan saberes originarios en el contexto urbano?

4. ¿Cómo adoptan estos saberes en su vida teniendo en cuento lo personal, familiar y social?

Por otra parte, se revisaron escritos de naturaleza grupal y documentos audiovisuales y fotográficos ${ }^{5}$. El análisis se desarrolló a partir del rastreo de archivos disponibles su clasificación respectiva. Se seleccionaron los documentos pertinentes para la investigación y se procedió a su análisis. Finalmente, se hizo lectura cruzada y comparativa de los documentos en cuestión. Debido al proceso de recuperación cultural generado por la comunidad, también se recurrió a la indagación y consulta de archivos históricos, además de diversas fuentes bibliográficas; esto permitió identificar elementos históricos relevantes en la configuración imaginaria emergente.

\section{Estructura de análisis: Triangulación}

Dadas las pautas del diseño metodológico se realizó la triangulación de las técnicas de registro señaladas y usadas de forma coordinada y complementaria,

5 Las fotografías utilizadas en la investigación son registros visuales compartidos por la comunidad. 
como estrategia metodológica que favorece una interpretación global, variada y fiable del fenómeno social a estudiar desde distintas perspectivas que enriquecen el proceso de investigación (Sandoval, 2002). De modo que la triangulación es entendida como un proceso de uso y contraste de varias estrategias, que permiten comparar y complementar los resultados obtenidos de cada una de éstas desde diferentes ángulos (Denzin; citado por Arias, 2009).

La elección de esta estrategia metodológica se halla en la indagación de patrones de convergencia, al dotar a los resultados en su interpretación y construcción de mayor relevancia y fiabilidad. Al utilizar una sola estrategia en el proceso de obtención de información, se considera que los estudios son vulnerables a sesgos y fallas metodológicas inherentes a cada estrategia como menciona Arias (2009). Se puede afirmar que esta metodología tiene un nivel de confiabilidad considerada admisible y cuenta con validez de constructo. De acuerdo con lo anterior, la figura 1 muestra el proceso de triangulación en la investigación:

Figura 1. Triangulación

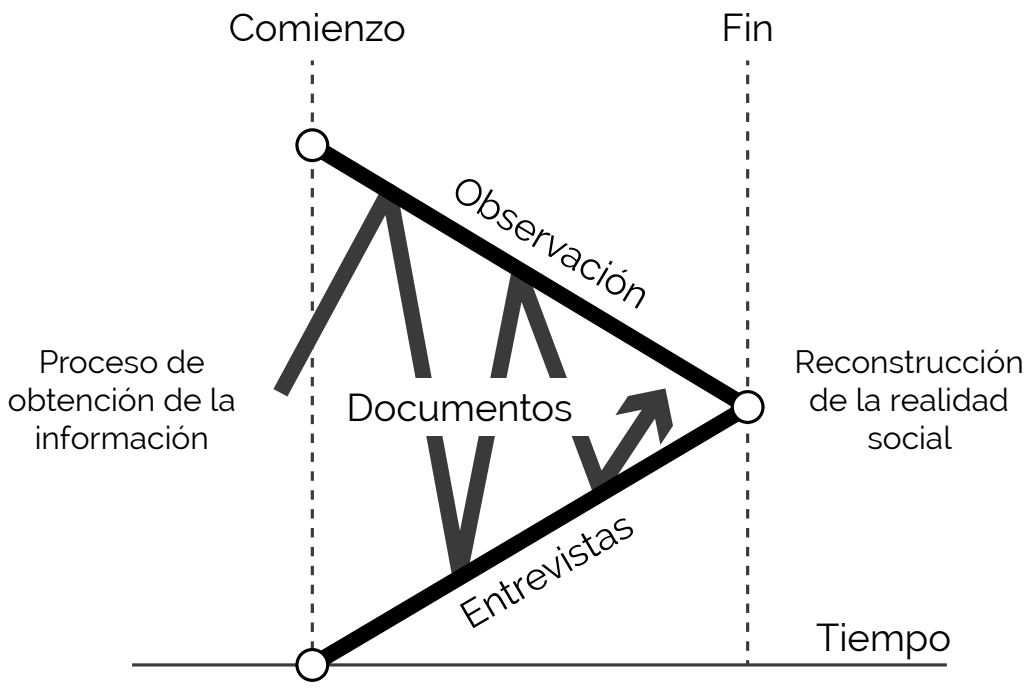

Fuente: Del Rincón (1995)

Una vez recopilada la información se dio paso a la triangulación de datos, que consistió en el estudio de elementos fundantes que permitieron verificar, comparar o confirmar una interpretación del fenómeno en estudio (Patton, 2002). Finalmente, se consideró que la triangulación confiere al estudio profundidad, complejidad y permite dar grados variables de consistencia a los hallazgos. 


\section{Resultados}

\section{La ciudad entre lo instituido y lo instituyente}

$\mathrm{Al}$ considerar la ciudad como escenario de efecto imaginario se comprende la producción subjetiva (social y cultural) de los individuos, sus percepciones a partir de lo manifiesto y latente de sus discursos y prácticas. Con base en ello, se interpretan las significaciones imaginarias para dar cuenta de lo instituido e instituyente de la ciudad, como institución imaginaria central. Para Castoriadis (1989) las significaciones imaginarias son producciones de sentido creadoras de representaciones, afectos e intenciones de una sociedad, desde las cuales los sujetos despliegan un universo de sentido que configura un imaginario social, otorgándole a la ciudad, como institución imaginaria, una red de sentido en permanente construcción que la configura y sustenta.

En este horizonte de interpretación, las sociedades se entregan a una invención permanente de su propia realidad y consolidan representaciones colectivas que expresan siempre en algún punto un estado del grupo social, su estructura, ideas y formas en que se relacionan los sujetos que las crean. De manera que cada sociedad genera su propio mundo dotado de significaciones al forjar las representaciones que determinan y definen lo que es real y lo que no, qué tiene sentido y qué no; al respecto, dice Baeza (2000) que los imaginarios son constructores de realidad(es) que al designar el campo de lo real interviene en las representaciones de los sujetos.

Entonces, aludir al imaginario social implica referirse a un espacio social históricamente construido, visible en el modo en que los sujetos imaginan su existencia, el tipo de relaciones que mantienen y las prácticas comunes vivenciadas, como señala Taylor (2004, p. 37); esto involucra una concepción colectiva del entorno social que permite unas prácticas comunes y un sentido compartido de legitimidad. De manera que el mundo social se constituye y articula a través de una serie de significaciones, que durante la observación participante se evidencia están instituidas, pero que a su vez forja lo instituyente, una nueva perspectiva de acuerdo con los postulados de Castoriadis (1989).

Lo anterior se establece como referencia para interpretar las percepciones, en tanto que las personas entrevistadas expresaron una reflexión crítica sobre la crisis del planeta tierra y la presente civilización, con respecto a una ideología de progreso esencialmente materialista, de explotación y gasto de recursos energéticos y naturales. Al examinar algunos elementos, se visualiza una constante reflexión sobre las concentraciones urbanas que en un tiempo fueron consideradas la expresión más perfecta de los beneficios del desarrollo económico capitalista y 
hoy son estimadas como la manifestación más acabada y brutal de la crisis actual (Vega, 2009).

Ahora, las ciudades promesa del progreso, del desarrollo civilizatorio comienzan a ser cuestionadas, puesto que las grandes concentraciones urbanas en su desmedido crecimiento, de acuerdo con Rodríguez (2013), provocan segregación social, privatización y utilización irracional de recursos, servicios y espacios, bajo la ilusión de crecimiento. De este modo, el contexto urbano y, desde luego, las dinámicas propias del sistema capitalista, hacen que la ciudad sea percibida como un espacio que se encuentra en un momento coyuntural, como se expresa los siguientes testimonios:

"Estamos viviendo en un caos, cada vez hay menos oportunidades para la gente, cada vez es más inhumano, cada vez se pierden más el valor del ser. La contaminación, tráfico, el afán, la indiferencia [...] El bienestar que nos ha enseñado esta sociedad es asociado a comodidades, lujos, cada vez el sistema nos crea más necesidades; entonces es quien tiene más, quien gana más” (Diana Correal).

"Todas las ciudades sin duda alguna tienen un desarrollo y un colapso; y la ciudad de Bogotá en los últimos años ha generado mayor desigualdad, barrios marginales, poca inversión, mucha corrupción; y esto ha hecho que la ciudad y ese ideal de la Edad Moderna donde las ciudades eran la cuna de las civilizaciones se van desboronando. El colapso de la ciudad todos los días en los trancones, la desigualdad económica, la gente sobreviviendo, el maltrato animales, la contaminación de los ríos, del aire. Esto hace que la ciudad comience a cambiar, porque la civilización que se pensaba ya no está [...] La civilización lo decía Enrique Dussel es el eslabón de la Modernidad en donde todos debían estar, en las industrias, en esa materialidad occidental que se impuso; pero vemos que a la gente le falta lo más valioso que es la felicidad" (Leonardo Guerrero).

"En nuestra forma de vida, nuestro pensamiento ha sido resistir hacia la forma de vida que muestra la ciudad. La idea es tratar de hacer un cambio, llevar una palabra, sensibilizar a la gente. Mejorar la calidad de vida de nuestras familias, conservar la cultura, alimentarnos diferente, retomando las culturas ancestrales, vivir de forma diferente, como comunidad" (Alberto Claro)

6 Entrevista realizada a los integrantes de Intillay Zhysua, entre los meses de marzo y junio del 2015 Debido a las características del presente documento, no serán citadas todas las entrevistas efectuadas. 
"El contexto de la ciudad nos lleva a mantener una forma de vida en constante resistencia ante todo lo que nos presenta, urbanización, falta de espacios naturales, pérdida de la identidad, imposición de un sistema capitalista que nos obliga a producir todos los días para mantener ese mismo sistema que nos somete. La ciudad es el espacio donde nos desenvolvemos; pero que resistimos diariamente [...] Hemos decidido adoptar ciertas prácticas que nos permiten acercar a un modo de vida diferente en la ciudad, la comunidad nos permite reconocernos dentro del contexto urbano, nos convocan a vivir bien” (Diana Salamanca).

En las percepciones manifestadas, se identifica la reflexión de una realidad instaurada y consolidada socialmente, enmarcada en el colapso de sociedades capitalistas como la ciudad de Bogotá. Lo anterior, se analiza y categoriza como la constitución de un imaginario instituido, siguiendo la teorización de Castoriadis (1997), en la cual se configuran unas percepciones como producto de la relación entre los individuos y la sociedad; esto es visto por ellos como un aspecto que funciona para conservar un orden social determinado. Lo anterior, es cuestionado por la comunidad, en tanto piensan que dicha situación tiende a permanecer e instituirse, al reproducir y mantener un sistema capitalista que regula y orienta los estilos de vida de los sujetos.

Como señala Castoriadis (1997), la reflexión de lo instituido socialmente, lleva inmersa la posibilidad de creación de un escenario en el que las personas modifiquen la realidad que los envuelve y creen un otro inédito, otra alternativa de existencia. Con respecto a ello, se identificó que la comunidad establece una cohesión interna desde la reflexión misma de lo instituido en dirección a lo instituyente, al generar la alteración del orden social instaurado que se evidencia en la creación de modos de vida alternativos.

Conviene subrayar que el concepto comunidad, engloba relaciones sociales y, a la vez, se refiere a un espacio delimitado por los sujetos (Bello, 2004, p. 106); por tanto, describe una entidad socialmente construida sobre la base de elementos objetivos como historia común y formas de cooperación que la establecen como aspecto de cohesión e identidad a través de procesos de subjetivación. La comunidad, entonces, es un hecho socialmente construido por los integrantes de Intillay Zhysua; ello, sustenta los procesos de identificación.

Según la perspectiva teórica de Terrén (2002), el tipo de construcción cultural que señala el discurso comunitario, permite mostrar la dinámica de lo que podría denominarse como la etnicidad vivida, recalcando que ésta no se define por la particularidad cultural objetiva sino por la construcción social y las estrategias que los sujetos utilizan para otorgarle sentido a su existencia en un grupo de pertenencia; de ahí que se reconozca que la etnicidad se desarrolla, fundamentalmente, a través de la interacción social y contribuye a la formación de 
una identidad en la que podrían aparecer aspectos objetivos, aunque no es algo exclusivo que determine la existencia o no, de este tipo de identificación. Como estrategia de estudio la investigación toma como Unidad de Análisis un espacio consolidado por la comunidad, a partir del cual se examinan dos prácticas en particular.

\section{Hacia una perspectiva histórica y mítica de la ciudad}

La investigación muestra que la consolidación del imaginario instituyente sobre la ciudad de Bogotá, se afianza a partir de la recuperación de un acervo de conocimientos y elementos vernáculos, en un proceso de re-significación que busca conocer y recuperar los saberes de la Cultura Mhuysqa que habitaba antiguamente la ciudad. Dicho proceso se materializa en un espacio de encuentro denominado Kuqa en lengua mhuysqhubun: Seminario o Centro de enseñanza (Escribano, 2007, p. 10), en el que Intillay Zhysua estudia y analiza, desde disciplinas como la arqueología, la linguiística, la topografía, la historia, entre otros elementos constitutivos de dicha cultura.

Y cuando se habla de experiencias de re-significación, estas deben ser concebidas como un "proceso social que afecta cualquier asunto de la vida en comunidad” (Molina, 2013, p. 44), que implica un ejercicio de trasformación, de volver a significar aspectos relevantes en las formas de vida y contenidos que dan cuenta de una subjetividad e identidad.

Además del análisis documental, se destaca la recuperación de narraciones mito-cosmogónicas a través del ritual de la palabra en ceremonia de Mambeadero, en que los participantes sentados en círculo escuchan las historias para comprender el sentido y las connotaciones espirituales presentes en los mismos.

\section{Mhuequeta: Pradera de campos cultivados}

La interpretación de Intillay Zhysua sobre el territorio, está mediada por la recuperación de elementos históricos en relación con un pasado Mhuysqa, analizados a partir del rescate de costumbres, nombres en lengua Mhuysqa (Mhuysqhubun) y la práctica de determinados rituales. Debido a las características de la investigación, se considera pertinente hacer visible los elementos que permiten dicho proceso de recuperación.

En el trabajo de campo efectuado se identificó la revisión documental de varios estudios, entre estos, los del antropólogo Beltrán (1993) y la historiadora y lingüista Escribano (2000), quienes establecen una descripción del medio geográfico de variada y exuberante naturaleza donde habitaban los aborígenes; además de coincidir en indicar que el nombre otorgado al territorio por los Mhuys- 
qa era Mhuequeta, es posible apreciar que este aspecto es valorado y apropiado por Intillay Zhysua al identificar y nombrar a Bogotá con el calificativo otorgado por los antiguos, lo cual permite pensar que se pretende reivindicar dicho nombre y, con ello, la cultura Mhuysqa.

Así mismo, se identificó que la interpretación y apropiación del territorio no solo se efectúa desde su designación, sino que también presenta una praxis comunitaria, la cual se sustenta en hallazgos que revelan la existencia de áreas del territorio dedicadas a la astronomía.

Frente a este aspecto, la comunidad analiza las investigaciones de Izquierdo y Morales (2006), quienes señalan registros de áreas del territorio colombiano dedicados a la orientación y alineación astral, entre estos, la Sabana de Bogotá. La comunidad también afirma que el relieve montañoso del altiplano facilitó la labor astronómica al ser utilizados como puntos de referencia, pues en perspectiva hacia los cerros donde se encuentra la Plaza de Bolívar, dicen que los antiguos observaron en la fecha solsticial de diciembre, la salida del sol sobre la montaña de Guadalupe y el solsticio de Junio tras Monserrate; en tanto, en los amaneceres de los equinoccios de marzo y septiembre, el sol se asoma en el punto medio que forman las dos montañas:

Figura 2. Alineamientos solares desde la Plaza de Bolívar en Bogotá

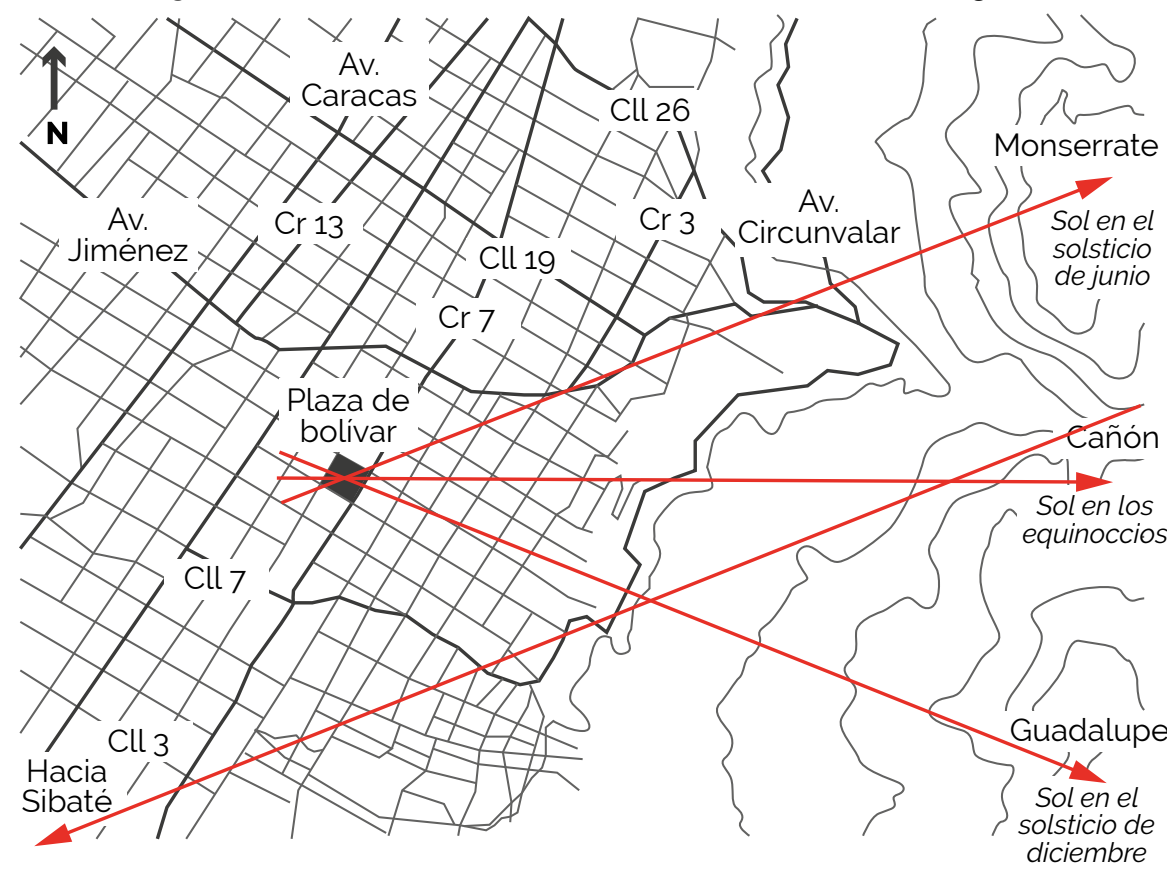

Fuente: Instrumental astronómico prehispánico en la cultura Muisca (Izquierdo y Morales, 2006, p. 170) 
Lo anterior sugiere que existieron lugares en Bogotá utilizados para hacer observaciones astronómicas en dirección a los cerros orientales. También se estudian las exploraciones del arquitecto Santos (2014), quien señala que durante el proceso de evangelización y extirpación de lugares que denotaban importancia para las culturas originarias, la invasión europea construyó centros de culto religioso para eliminar y ocultar cualquier vestigio de los sitios sagrados y así establecer una nueva simbología (pp. 290-300). Lo anterior es analizado por la comunidad en el contexto de la ciudad, mientras que en el punto de observación del recorrido del sol, en la esquina nororiental de la Plaza de Bolívar, se encuentra de espalda a los cerros de Monserrate y Guadalupe, la Catedral Primada de Colombia que obstruye la actividad astronómica.

El estudio permitió evidenciar que los integrantes de Intillay Zhysua asisten cada solsticio y equinoccio a la Plaza de Bolívar, para contemplar los astros con la intención de re-significar este espacio y recuperar un acontecimiento o acto vivido por los Mhuysqa, como se expresa a continuación:

\begin{abstract}
"Nosotros vamos a la Plaza de Bolívar el 21 de diciembre y el 21 de Junio porque allí antiguamente era un observatorio solar para los muiscas, entonces nosotros tratamos de recuperar esa práctica y cada año miramos el recorrido del sol y recibimos su luz sobre Guafa y Tensaca. Es la renovación de la energía solar a nivel de los ciclos de los solsticios y en el caso de la luna los equinoccios" (Alejandra Restrepo).
\end{abstract}

"Vitalmente es un observatorio astronómico la Plaza de Bolívar, pues como se impuso un poder en el momento de la conquista, nos quedó la herencia y la memoria de lo celeste, el lugar donde podemos ver los equinoccios y los solsticios. Es un punto de encuentro astronómico, es el recordar nuestro pasado” (Óscar Guerrero).

Junto a esta práctica, es posible darse cuenta de que los espacios se vuelven a nombrar de acuerdo con su origen, pues la comunidad nombra a los cerros por su denominación originaria: Cerro de Guadalupe: Guafa; Montaña de Chusque (Mhuysqhubun) y Cerro de Monserrate: Tensaca; Montaña Cerrada. Apropiar la ciudad desde esta perspectiva es una práctica Mhuysqa valorada y apropiada comunitariamente que constituye un sustento ontológico al permitirles construir una nueva representación en la cual la ciudad toma otro sentido, en tanto se reitera y vigoriza una práctica ritual vivenciada por los antepasados. Se identifica así, una percepción particular de la urbe desde elementos vernáculos que configura unas maneras de ser, teniendo presente que los aspectos subjetivos construidos están mediados por un proceso de re-significación, de intervención 
social que termina por promover transformaciones en la manera en que se construye y vive la realidad (Molina, 2013).

Figura 3. Celebración Solsticio 2010, Plaza de Bolívar de Bogotá

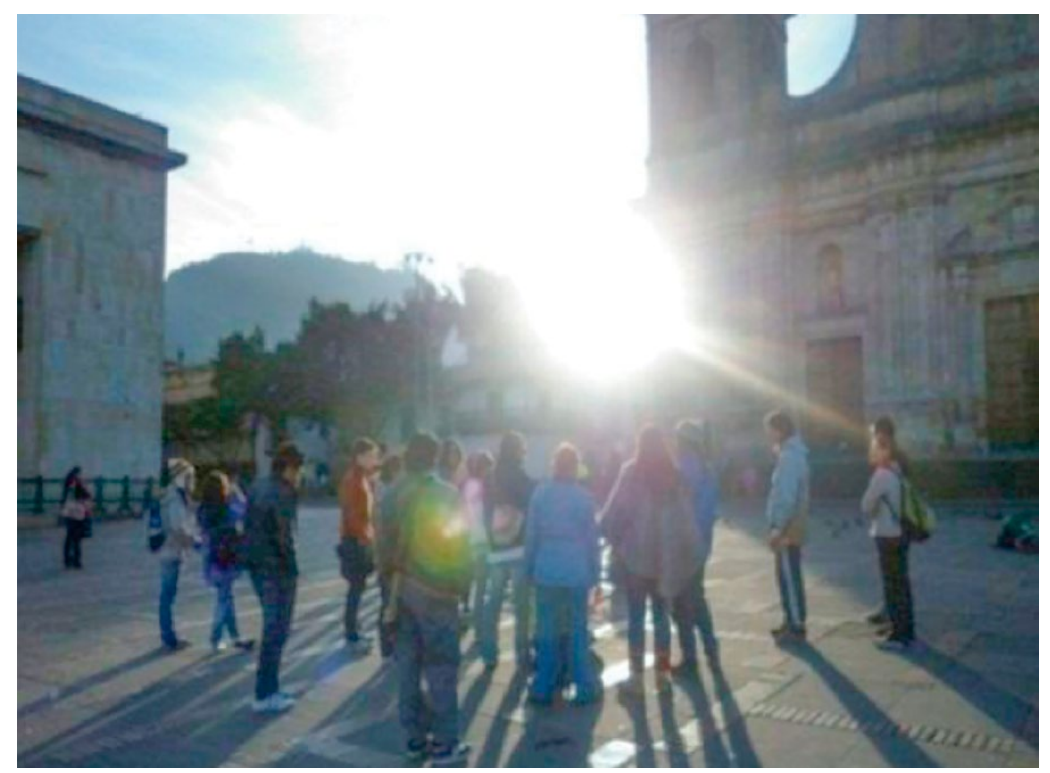

Fuente: Archivo fotográfico Intillay Zhysua

Para que una sociedad exista y se mantenga, para que pueda asegurarse un mínimo de cohesión y de consenso, es imprescindible que los sujetos crean en la superioridad del hecho social sobre el hecho individual, que tengan en fin, una conciencia colectiva, un sistema de creencias y prácticas (Baczko, 1984, p. 21). De acuerdo con lo anterior, en Intillay Zhysua se presenta a la vista la afirmación de una conciencia de pertenencia a una comunidad, la cual exalta a la vez, una manera de pensar materializada en unos modos de acción que reconstituyen y perpetúan las creencias necesarias para que se dé el consenso social; de igual manera, se identifica el reconocimiento de la común-unidad como forma de vida, en que la memoria se establece como mecanismo cultural para fortalecer el sentido de pertenencia a la comunidad (Jelin, 2002, p. 10). 


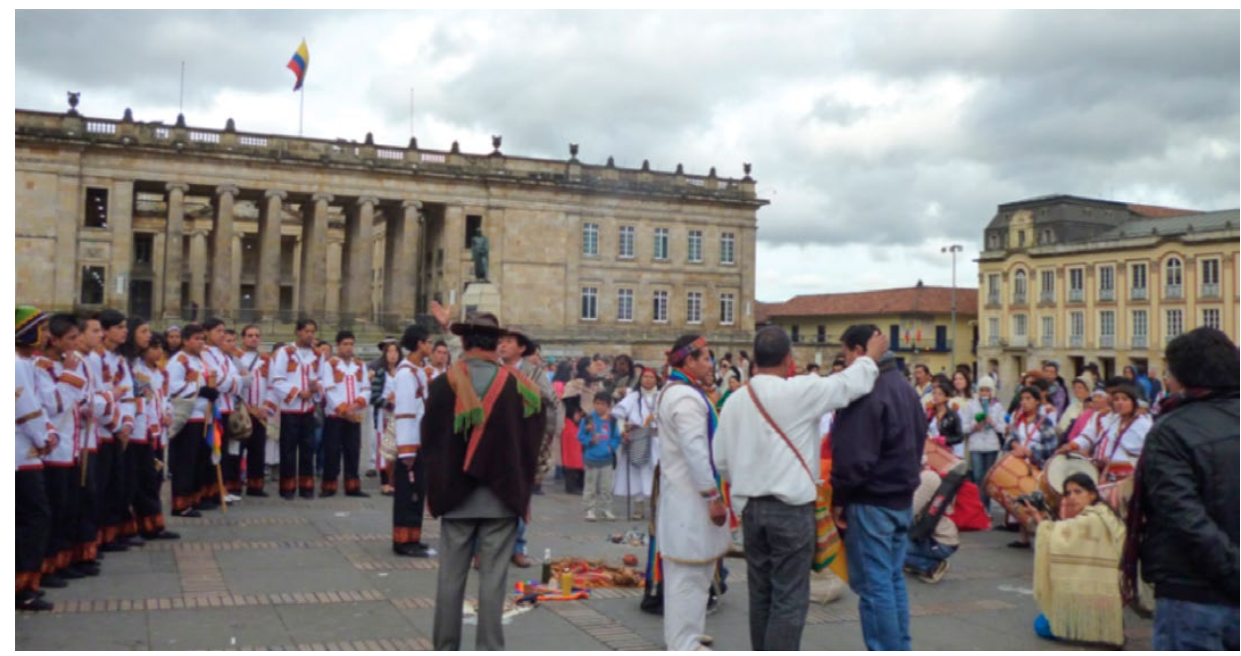

Fuente: Archivo fotográfico Intillay Zhysua

Junto al análisis documental efectuado la narración mitológica, se presenta como elemento central para Intillay Zhysua el reconocimiento de que en la antigüedad, con explicaciones filosóficas y científicas sobre el mundo, se entró en competencia con las explicaciones míticas consideradas como un relato con la pretensión de ser explicación histórica, quedando así como una creencia extendida pero falsa; además de ser juzgado desde los parámetros de la racionalidad (Eliade; 2001).

Es importante mencionar que el mito es un elemento vital de la civilización humana; no es una explicación intelectual ni una imaginería del arte, sino la constatación de una realidad que devela a partir de los principios de vida, actos rituales, acciones morales e incluso, actividades prácticas. En muchas sociedades, "lo vivido como real no es la temporalidad histórica, sino el tiempo mítico que remite permanentemente, en rituales y repeticiones, a un momento fundacional, original" (Jelin, 2002, p. 24); en este sentido, el mito enriquece la realidad cultural que contempla la existencia de un esquema de creencias cumpliendo una función integradora que rige la vida de los sujetos, frente a lo cual integrantes de Intillay Zhysua expresan:

"El mito en la actualidad tiene una resignificación, pues históricamente ha sido configurado desde una historia basada en la imaginación, en algo que no existe o fue una interpretación a partir de un lenguaje muy fantástico. Para nosotros 
el mito si es imaginación, pero una imaginación que potencia, para recrear la realidad de una forma diferente" (Yeimy Ibarra).

"El mito nos ha servido para contar historias, las cuales nos ordenan en la forma de actuar, pensar, sentir [...] Por medio del mito se ha buscado, más que crear una ley, permitir que cada persona asuma desde el mito un conocimiento de una perspectiva de ser, de actuar y vivir" (Leandro Sanabria).

Podría decirse que para la comunidad el mito es una explicación metafórica de la realidad, el cual tiene implicaciones históricas, sociales y ontológicas, en tanto que ellos expresan que el interés no solo es hablar del mito, sino hablar de la necesidad de comprender mitológicamente el mundo con la intencionalidad de compartir y recordar los relatos que circundan la cosmovisión de la cultura Mhuysqa. Uno de los mitos compartidos considerado fundamental para esta investigación es la narración sobre Bochica, legislador de los Mhuysqa citado a continuación ${ }^{7}$ :

"Padre Bochica en las historias mitológicas Mhuysqa aparece como el primer hombre de formación en la tierra, quien recibe el nombre de Cuza "semilla de la energía en negativo" y junto con la primera mujer Furachogua "semilla de la energía en positivo", se unen permitiendo la procreación del hombre, y posteriormente del pueblo Mhuysqa $[\ldots]$. Luego hombres y mujeres caminamos por la tierra. Como nómadas, vestíamos con pieles de animales, comíamos frutos, nuestra vivienda eran los abrigos rocosos y nos encontrábamos en torno al fuego. No éramos aún comunidad. Posterior a su primera aparición, Cuza evoluciona y se hace sabio, recorre la tierra para entenderla, desde los animales, las plantas, los minerales, desde todas sus formas. Un día esplendoroso entró por los abrigos rocosos del hoy Tequendama, en ese momento recibe el código de Nemqueteba "capitán del bosque" que quiere decir quien es instruido por la naturaleza y los felinos en su andar, en su caminar.

Vestido con mantas de algodón blancas, de cabellos y barba largas y canosas, con un broche de oro sobre su hombro derecho y montado sobre una llama, congrego a los Mhuysqa mientras caminaba de sur a norte, trasmitiendo su conocimiento a su propia especie, a sus propios hijos; aparece entonces un proceso formativo de él como el creador de las formas, de las alquimias físicas y químicas de la

\footnotetext{
7 Narración mitológica compartida en ceremonia de Mambeadero entre los miembros de Intillay Zhysua, la cual fue trasmitida por los abuelos y sabios de la Cultura Mhuysqa.
} 
naturaleza. En su andar se encontró con un Mhuysqa que llevaba afanosamente unas piedritas de oro, su nombre era Piracá. Esté se tropezó dejando caer las piedritas al suelo y en ese preciso momento Nemqueteba le observa y le dice: ¡deja ahí, y vuelve en 20 días! Así fue, a los 20 días una mata de maíz crecía.

Entonces, Nemqueteba dijo a Piracá: De ahora en adelante será tu alimento sagrado. Vivirás, vestirás y serás como él. De esta manera Nemqueteba cambia su código a Bochica, enseñando la agricultura. Además del trabajo con el oro, la arcilla, los tejidos en algodón, la lengua Mhuysqhubun, la estructura de la familia y la comunidad. Comenzó a caminar, a enseñar y dejar organizada la comunidad; el primer territorio al que llegó lo llamó Suacha "sol varón" ya que Bochica recordaba el culto al sol, de ahí su broche en el hombro derecho. A partir de su peregrinación los pobladores dejaban a su comunidad un nombre que recordara el caminar del primer abuelo Bosa: El círculo, el cercado. El número 2, lugar donde muere su llamita.

Cota: el de cabello largo, haciendo mención a su cabellera.

Funza: Varón poderoso.

Fontyba: Capitán poderoso.

Zhuba: el reflejo de mi rostro, mi compañero.

Engatyba: Capitán de lo ameno.

Techotyba: Buen boquerón del capitán.

Suacha: Sol varón

"Es así que Bochica vive 400 años sobre la tierra, 400 edades que son reencarnaciones sucesivas para poder llegar al estado sagrado y sublime del padre de la humanidad, primer maestro ascendido, el padre barbudo cósmico, quien después de entregar todo su conocimiento decide retornar a la laguna madre de Iguaque con la primera mujer y convertirse en los dos maestros espirituales Bochica y Batchue" (Carlos Martínez).

En la narración citada, se identifican algunos hechos históricos concernientes al caminar de Bochica que establecen una relación con un sistema de organización social basado en labores como el tejido en algodón y la siembra del maíz. Uno de los aspectos más sobresalientes de la narración, es la conservación de aspectos constitutivos de esta cultura que expresa, realza y codifica creencias de un conocimiento antiguo; así mismo, se presenta la designación en lengua mhuysqhubun de los lugares en que este personaje caminó, y actualmente son localidades de Bogotá y municipios aledaños. 
Esta designación muestra una nueva significación de los territorios a partir de las enseñanzas del demiurgo, lo cual es apropiado por la comunidad, constituyéndose en una percepción de la realidad en el sentido y significado que se le confiere al espacio de la ciudad, mediado por la experiencia vivida en el Kuqa de investigación, desde de un referente histórico construido a partir del análisis documental señalado anteriormente y un aspecto mito-cosmogónico presente en la narración oral, permitiéndoles construir una percepción sobre el espacio plasmado en las figuras $3 \mathrm{y}$ 4:

Figura 5. Mhuequeta: Pradera de los campos cultivados. Kuqa de investigación año 2011

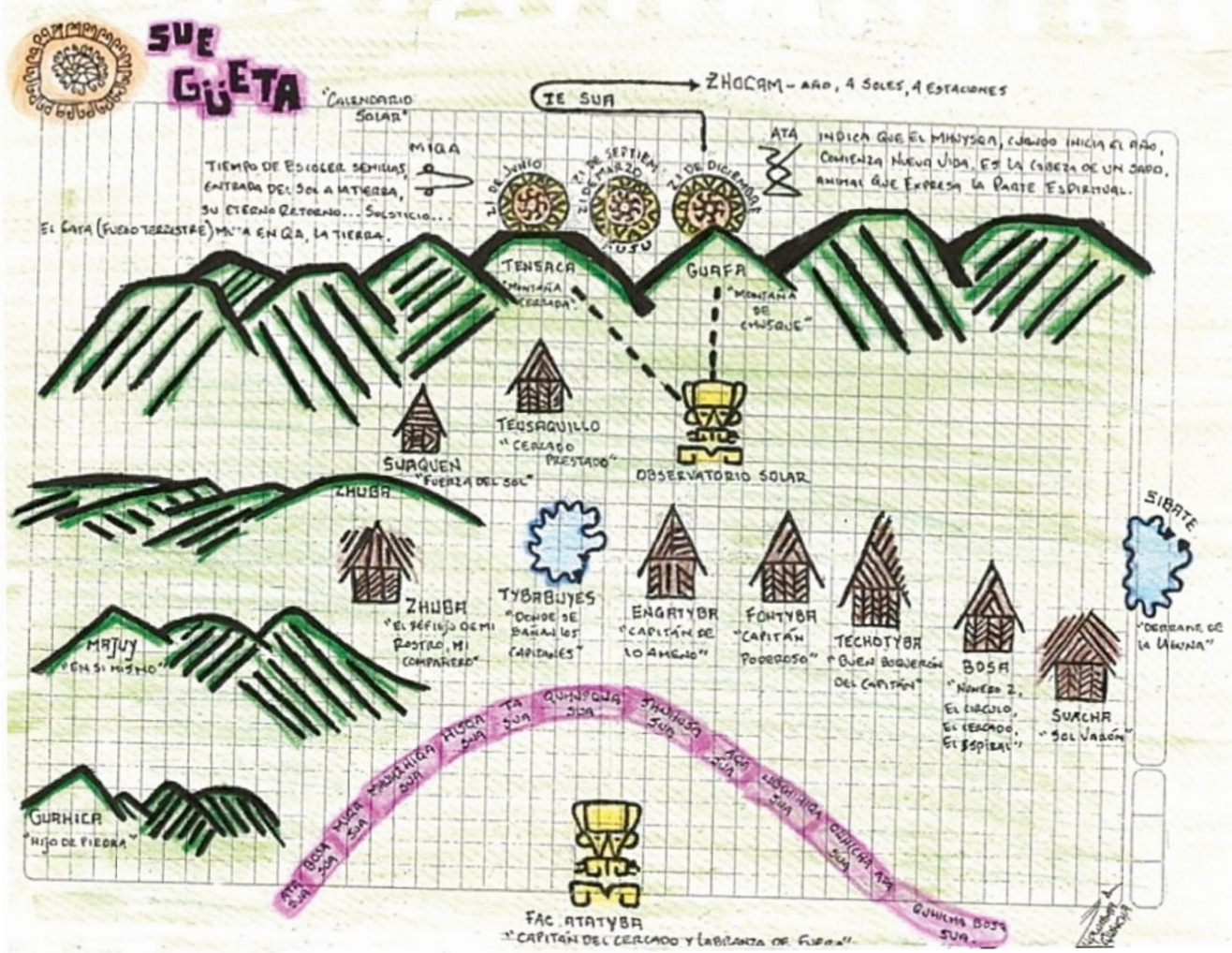

Fuente: Archivo documental Intillay Zhysua 


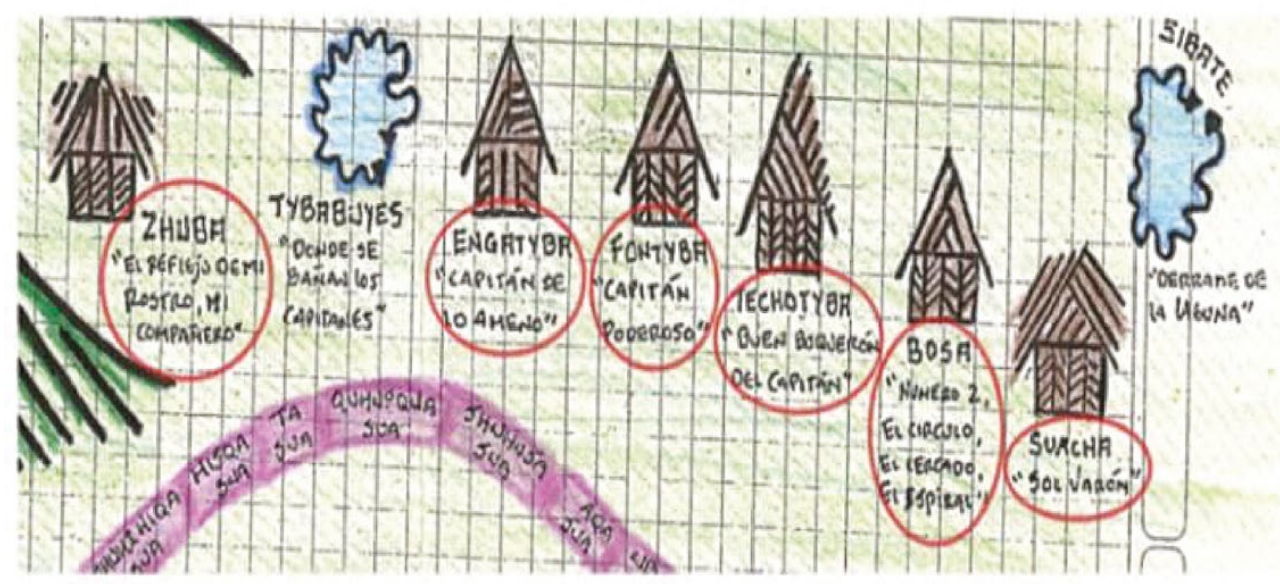

Fuente: Archivo documental Intillay Zhysua

Las figuras 3 y 4 muestran la percepción que tiene la comunidad de la geografía de la Sabana y algunas construcciones del espacio urbano, en el cual se puede identificar la pretensión de recuperar rasgos históricos y revalorar algunas prácticas en el territorio, pues se plasma en el esquema los cerros de Monserrate y Guadalupe, Tensaca y Guafa respectivamente, en dirección al observatorio astronómico; lo anterior se analiza como un elemento constitutivo del universo simbólico y las concepciones de mundo construidas por Intillay Zhysua. Otro aspecto relevante que se observa en la narración mitológica y en el esquema citado, es el reconocimiento de algunas localidades de la ciudad a partir de la comprensión y el rescate de la toponimia, nombrándolas a través de la designación dada por Bochica.

La relación establecida entre los nombres originarios y los acontecimientos que ocurrieron en estos, configuran unas representaciones del espacio fundamentales para entender los procesos de apropiación del territorio; esto, debido a que la ciudad imaginada genera unos nuevos usos sociales de ésta, al exponer los efectos simbólicos que producen las condiciones físicas naturales (cerros orientales) y físicas construidas (Plaza de Bolívar) definidas en geografías transmisoras de sentido (Silva, 2006).

En este sentido, los significados que se le atribuyen a la ciudad le confieren una impronta a los usos y a las prácticas que se desarrollan en ésta, asociados a un pasado vernáculo en que aparecen iniciativas que fundan nuevas cohesiones sociales y culturales, al tratarse de acontecimientos que se establecen en estruc- 
turas de sentido donde lo ritual y lo mítico ocupan un lugar privilegiado como aspectos claves en la activación de la memoria (Jelin, 2002, p. 18) y representación del pasado. Se identifica que el acto de rememorar presupone, tener una experiencia pasada que se revive en el presente y permite pensar que no solo se trata de la conmemoración de un acontecimiento mítico, sino de su reactualización al volver a significar unos modos de actuar, lo cual se considera como un hecho o práctica social, en tanto que modifica y establece una nueva realidad.

Así pues, los resultados de este estudio evidencian la existencia de discrepancias sobre el imaginario instituido de la ciudad creado hegemónicamente y el imaginario que ha consolidado la comunidad Intillay Zhysua. Tal discrepancia no se presenta como una situación de rivalidad o pugna, sino en un proceso constante de reflexividad y dilucidación crítica con respecto a lo instituido socialmente. Conforme a ello se halló que la configuración de un imaginario instituyente, en donde los sujetos al vivir en una sociedad dada, la interpretan dando lugar a la emergencia de nuevas significaciones y a la creación de otra realidad que diferiré de lo establecido.

De acuerdo con los resultados de este estudio se infiere que la configuración imaginaria instituyente consolidada por la comunidad, se sustenta en procesos de recuperación y re-significación de los saberes de la Cultura Mhuysqa, a partir de un conjunto de prácticas milenarias y significados socialmente compartidos que les ha permitido hacer efectiva una nueva perspectiva de la realidad.

Los hallazgos de la investigación también permiten observar que la constitución del imaginario sobre la urbe está ligado al sentido que Intillay Zhysua le confiere al territorio, como escenario transmisor de sentidos. Es pertinente señalar que la ciudad vivenciada, es resultado de las significaciones que ellos construyen desde elementos históricos, geográficos y culturales rescatados y re-significados, los cuales les posibilitan consolidar sentidos de identidad y un proceso de recuperación de la memoria, en que el pasado se convierte en un principio de acción para el presente (Jelin, 2002) producto de la afirmación de un sentimiento de pertenencia a la comunidad.

Ahora bien, la ciudad como creación y producto social sobre el que se adhieren los elementos de la estructura social, expresa como lo muestran los resultados, la visión de la comunidad a partir de una mirada diferencial posible de categorizar en una marca territorial que se usa e inventa en la medida que las personas lo apropian, lo nombran y viven dándole, a su vez, un nombre que evoca su uso imaginario (Silva, 2006, p. 61), para el caso como se muestra, la ciudad del altiplano circundada entre montañas: Mhuequeta, cuya representación es una apropiación, sostenida por un conocimiento y una práctica que procura preservar un pensamiento que afecta los modos de simbolizar aquello que se conoce como realidad. 
Es factible establecer, de acuerdo con lo anterior, que las relaciones con el territorio operan como la representación de una relación de exclusividad e identidad a través de un proceso de subjetivación de factores objetivos, en este caso y según los resultados, el territorio como factor de identidad, de pertenencia común esencial para la configuración imaginaria. Lo anterior es fundamental en tanto que la ciudad transmite una historia y una cultura que no siempre es la misma para sus habitantes, sobre todo cuando las poblaciones son cada vez más diversas por su origen y forma de vivir en las zonas urbanas.

Los resultados además enfatizan que el mundo histórico-social constituido por las significaciones imaginarias, se establece en un proceso de re-significación de prácticas y concepciones comunitarias que se apoyan en la identidad cultural presente en el territorio que pretende revalorizarlo, reinventarlo, al trascender en las construcciones de vida de los integrantes de la comunidad, dado que se identifica la exaltación de los aportes que realizan las relaciones rituales al interior del contexto urbano, que les permite apropiar la ciudad como escenario de ciertas prácticas en la emergencia de una nueva forma ontológica, un nuevo eidos diría Castoriadis (1997), un nuevo modo de ser (p. 5).

Según lo anterior y siguiendo a Molina (2013), el proceso de re-significación consolidado corresponde a una situación y condición deseada y éticamente valorada que alude a una responsabilidad tanto individual como social que describe los cambios y la trasformación de la construcción social de la realidad.

En este sentido se muestra que la interpretación sobre la ciudad responde a la construcción social de un imaginario, en la medida en que como señala Silva (2006) "los imaginarios sociales serían precisamente aquellas representaciones colectivas que rigen los procesos de identificación social, y con los cuales, los sujetos interactúan forjando unos modos particulares de comunicarse e interactuar socialmente” (p. 104). Conforme a ello, los imaginarios como constructos de sentido se constituyen en formas de vivenciar el presente, al articular la imaginación a los ámbitos de la vida social, en tanto que el imaginario creado de la ciudad, se entiende como la construcción mental elaborada con base en unas percepciones y experiencias que le otorgan otros sentidos y significados al espacio.

De acuerdo con lo anterior, ocurre que el pasado que tal vez parecía olvidado definitivamente, reaparece y cobra vigencia, a partir de cambios en los marcos culturales y sociales que impulsan a Intillay Zhysua a revisar y dar nuevo sentido a saberes y prácticas que no se habían significado. 
Figura 7. Comunidad Intillay Zhysua, Resguardo Mhuysqa de Cota-Cundinamarca.

Hytchana: Nuestra Tierra Enero de $2016^{8}$

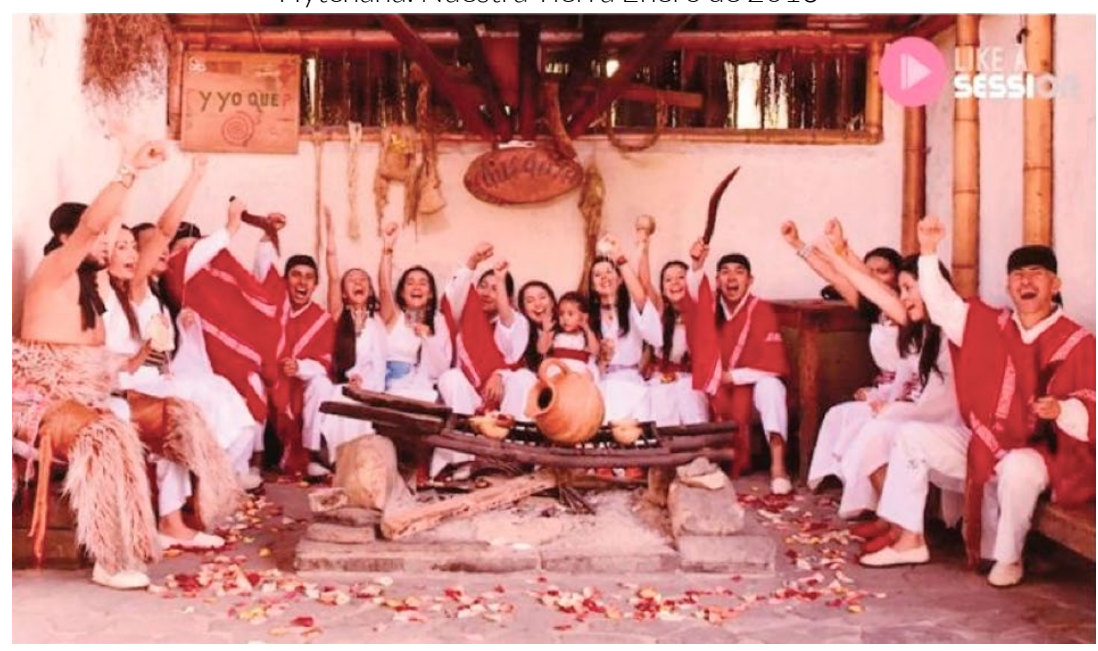

Fuente: Archivo documental Intillay Zhysua

\section{Conclusiones}

La investigación pone sobre la escena actual las formas de representación que surgen en la sociedad y los modos de vivir y contar la ciudad que generan la constitución y emergencia de lo imaginario. Con ello, se reconoce la existencia de ciertas prácticas y experiencias en las posibilidades de acción de los sujetos, que aportan al análisis y discusión sobre la existencia de diversas formas de ver el mundo, las perspectivas constitutivas de la acción humana y las realidades en emergencia (Piedrahita et al., 2010). Esto, a su vez, permite criticar la legitimación de formas coloniales de representación y subordinación de saberes, así como la redefinición y la modificación de las prácticas mismas de producción y circulación de conocimientos.

Lo anterior, es imprescindible para comprender e interpretar los aspectos que emergen en los procesos de auto-reconocimiento y conformación de grupos de pertenencia; además, resalta la posibilidad de identificación, de acuerdo con procesos definidos socialmente que aparecen bajo condiciones históricas determinadas y ciertos elementos culturales, lo cual juega un papel significativo 
en las formas contemporáneas de representación de lo social en el contexto específico de la ciudad de Bogotá.

En efecto, se considera una oportunidad fundamental en la construcción de otras formas de relación entre sujetos y saberes, además de constituirse como una posibilidad de redefinir las formas de pensamiento al establecer un diálogo con las múltiples maneras en que los sujetos observan, entienden el mundo. Se establece así un horizonte investigativo que busca promover ámbitos de investigación, metodologías y epistemologías que pretenden construir un conocimiento constitutivo de la realidad social, asumiendo el desafío de caminar hacia la recuperación y reivindicación de saberes orientados hacia el reconocimiento de la diversidad del ser humano.

\section{Referencias}

Arias, L. (2009). Interdisciplinariedad y triangulación en ciencias sociales. Diálogos, $10(1), 117$ - 136. http://www.redalyc.org/pdf/439/43913137005.pdf [Consultado el 10 de junio de 2018].

Baczko, B. (1984). Imaginario Social. Memoria y esperanzas colectivas. Paris, Francia: Editorial Payot.

Baeza, M. (2000). Los caminos invisibles de la realidad social. Ensayo de sociología profunda sobre los imaginarios sociales. Santiago de Chile: Ril Editores.

Bello, A. (2004). Etnicidad y ciudadanía en América Latina. La acción colectiva de los pueblos indígenas. Santiago de Chile: Naciones Unidas y CEPAL.

Beltrán, F. (1993). Los Muiscas, pensamientos y realizaciones. Bogotá, Colombia: Editorial Nueva América.

Castoriadis, C. (1989). La institución Imaginaria de la Sociedad. Barcelona, España: Tusquets Editores.

Castoriadis, C. (1997). Ontología de la creación. Bogotá, Colombia. Colección pensamiento crítico contemporáneo.

Del Rincón, D. (1995). Técnicas de investigación en Ciencias Sociales. Madrid, España: Dykinson. 
Echeverría, B. (2011). Crítica de la modernidad capitalista. La Paz, Bolivia: "Garza Azul” Impresores \& Editores.

Eliade, M. (2001). El mito del eterno retorno. Arquetipos y repetición. Buenos Aires, Argentina: Ed. Emecé.

Escribano, M. (2000). Cinco mitos de la literatura oral Mhuysqa o chibcha. Análisis profundo sobre una óptica de la semiótica estructural y una visión de la semiótica gnoseológica. Bogotá, Colombia: Samper Ediciones.

Escribano, M. (2007). Investigaciones semiológicas de la lengua Mhuysqa. Descifrado de los números del calendario lunar. Bogotá, Colombia: Antares Impresores Ltda.

Huanacuni, F. (2015). Vivir bien/Buen vivir. Filosofía, politicas, estrategias y experiencias de los pueblos ancestrales. Quito, Ecuador: Coordinadora Andina de Organizaciones Indígenas, CAOI.

Izquierdo, M. y Morales, J. (2006). Instrumental astronómico prehispánico en la cultura Muisca. En J. Lull (Coord.). Trabajos de Arqueoastronomía. Ejemplos de África, América, Europa y Oceanía (pp. 161-183). Valencia, España: Agrupación astronómica de La Safor.

Jelin, E. (2002). Los trabajos de la memoria. Madrid, España: España editores S. A. y Junio Siglo XXI.

Molina, N. (2013). Discusiones acerca de la Resignificación y Conceptos Asociados. Revista MEC-EDUPAZ, 1(3), 39-63. http://www.journals.unam.mx/ index.php/mecedupaz/article/view/36436/33013 [Consultado el 11 de marzo de 2018].

Montañez, G. (2001). Espacio y territorio. Razón, pasión e imaginarios. Bogotá, Colombia: Universidad Nacional.

Patton, M. (2002). Investigación cualitativa y métodos de evaluación. Bogotá, Colombia. Editores S. A. Tercera edición.

Piedrahita, C.; Jiménez, A.; Gómez, J.; Serna, A.; Oviedo, A.; Amador, J.; Díaz, C.; Borja, M. y Bravo, F. (2010). Desafío en estudios sociales e interdisciplinariedad. Bogotá. Colombia: Editores Antropos Ltda. 
Restrepo, E. (2004). Etnicidad sin garantías: contribuciones de Stuart Hall a los estudios de la etnicidad. En E. Restrepo. Teorías contemporáneas de la etnicidad Stuart Hall y Michel Foucault (pp. 36 - 72). Popayán, Colombia: Editorial Universidad del Cauca.

Rodríguez, I. (2013). Resignificando la ciudad colonial y extractivista. En Grupo permanente de trabajo sobre Alternativas al Desarrollo (Ed.). Alternativas al capitalismo colonialismo del siglo XXI (pp. 225-257). Quito, Ecuador: Ediciones Abya Yala.

Sandoval, C. (2002). Programa de especialización en teoría, métodos y técnicas de Investigación social: Investigación cualitativa. Bogotá, Colombia: ARFO Editores e Impresores Ltda.

Santos, R. (2014). Geografía sagrada del territorio Mhuysqa. En R. Sánchez y F. Mejía (Comp.). Cátedra de la memoria Mhuysqa. Homenaje al Maestro Guillermo Fonseca Truque (pp. 281-323). http://mensajesdelamadretierra. blogspot.com/ [Consultado el 16 de Agosto de 2018].

Silva, A. (2006). Imaginarios urbanos. Bogotá, Colombia: Arango Editores LTDA.

Taylor, C. (2004). Imaginarios sociales modernos. Barcelona, España: Paidós.

Terrén, E. (2002). La etnicidad y sus formas: aproximación a un modelo complejo de la pertenencia étnica. Papers 66, 45-57. https://ddd.uab.cat/pub/ papers/o2102862n66/02102862n66p45.pdf [Consultado el 20 de octubre de 2018 ].

Vega, R. (2009). Crisis de la civilización capitalista: Mucho más que una breve coyuntura económica. En J. Estrada (Comp.). Crisis capitalista económica, política y movimiento (pp. 61-101). Bogotá, Colombia: Espacio crítico Ediciones.

Zambrano, F. (2000). La ciudad en la historia. En C. Torres; F. Viviescas; E. Pérez (Comp.). La ciudad: Hábitat de diversidad y complejidad (pp. 122-148). Bogotá, Colombia: Universidad Nacional. 